\title{
Can local ablative techniques replace surgery for locally advanced pancreatic cancer?
}

\author{
Ulrike Heger, Thilo Hackert \\ Department of Surgery, Heidelberg University Hospital, Heidelberg, Germany \\ Contributions: (I) Conception and design: Both authors; (II) Administrative support: Both authors; (III) Provision of study materials or patients: None; \\ (IV) Collection and assembly of data: U Heger; (V) Data analysis and interpretation: Both authors; (VI) Manuscript writing: Both authors; (VII) Final \\ approval of manuscript: Both authors. \\ Correspondence to: Thilo Hackert, MD. Department of Surgery, University of Heidelberg, Im Neuenheimer Feld 420, 69120 Heidelberg, Germany. \\ Email: thilo.hackert@med.uni-heidelberg.de.
}

\begin{abstract}
In the treatment of pancreatic ductal adenocarcinoma (PDAC) the best chance at long term survival or cure has to date always included the complete surgical removal of the tumor. However, locally advanced pancreatic cancer (LAPC), about $25 \%$ of all newly diagnosed PDAC, is defined by its primary technical unresectability due to infiltration of visceral arteries and absence of metastasis. Induction therapies, especially FOLFIRINOX treatment, together with technical surgical advancement have increased the numbers for conversion to secondary resectability. Recent data on resections after induction therapy show promising, almost doubled survival compared to palliative treatment. Yet, around 70\% of LAPC remain unresectable after induction therapy, often due to persistent local invasion. As locally ablative techniques are becoming more widely available this review examines their possible applicability to substitute for surgery in these cases which we propose to group under the new term "Inconvertible LAPC". The need for defining this novel subgroup who might benefit from ablative treatment is based on the findings in our review that high-level evidence on ablative techniques for PDAC is largely lacking and the latest effective, harmonized treatment guidelines for LAPC are not often incorporated in these studies. The "inconvertible LAPC" label requires persistent unresectability after staging and induction therapy of LAPC according to current guidelines followed by liberal indication for aggressive surgical exploration at a center equipped for extended pancreatic resections. Ideally, this specification of a new, distinct patient group will also put it in the spotlight more, hopefully prompt more trials designed to generate robust evidence and optimize transferability of study results.
\end{abstract}

Keywords: Pancreatic cancer; locally advanced pancreatic cancer (LAPC); ablation techniques; irreversible electroporation (IRE); induction therapy

Submitted Sep 11, 2020. Accepted for publication Nov 04, 2020.

doi: 10.21037/jgo-20-379

View this article at: http://dx.doi.org/10.21037/jgo-20-379

\section{Background}

The claim "only steel can heal" describes not only traditional surgical self-confidence but also seems to sum up the clinical management of pancreatic cancer. While pancreatic surgery is risk-prone for perioperative morbidity and mortality complete removal of a pancreatic carcinoma remains the only chance at cure or longtime survival. Today's clinical pathways for pancreatic ductal adenocarcinoma (PDAC) distinguish resectable, borderline resectable (BRPC), locally advanced (LAPC) and disseminated stages $(1,2)$. About half of all newly diagnosed patients present with metastases and $15-20 \%$ are resectable upon diagnosis, according to data from the SEER database (3). As the benefit of neoadjuvant therapy in resectable tumors is still a matter of debate in spite of 
numerous trials and a few meta-analyses (4-7), international consensus guidelines recommend upfront resection for resectable PDAC outside of clinical trials. Five-year survival rates in the resectable group can reach around $20 \%$, in favorable subgroups exceeding $50 \%$ (8). In stage IV pancreatic cancer palliative chemotherapy with FOLFIRINOX is the current standard of care (9). In locally advanced stages without dissemination, however, recommendations are not so straightforward: BRPC and LAPC together comprise about $30 \%$ of all new cases and, semantically, some authors term BRPC the resectable subtype of LAPC. The distinction between BRPC and LAPC differs between guidelines which set different limits regarding the degree of venous and arterial involvement and in part CA 19-9 levels and performance status (3,10-14). Generally, though, LAPC is considered technically not completely resectable, whereas BRPC can be surgically removed by simultaneous resection of adjacent vasculature and/or multi-organ resections with an increased risk of microscopically positive margins. In BRPC many cohort studies as well as randomized controlled trials (RCT) to date have investigated possible benefits of preoperative (radio-) chemotherapy reflecting the emerging practice of choosing pretreatment over an upfront surgical resection in patients with an inherent risk of unresectability or microscopic incomplete resection [reviewed in (15-17)]. Consensus guidelines in Europe as well as the US recommend neoadjuvant treatment $(1,2)$ and a recent meta-analysis advocates for preoperative FOLFIRINOX specifically (16). LAPC in the unresectable sense is defined by the National Comprehensive Cancer Network (NCCN) as well as the International Study Group of Pancreatic Surgery (ISGPS) as: (I) no distant metastasis, (II) solid tumor contact with superior mesenteric artery (SMA) and/or celiac artery $>180^{\circ}$, (III) solid tumor contact with the first jejunal SMA branch and/or aortic involvement, (IV) unresectable superior mesenteric vein (SMV) and/or portal vein (PV) due to tumor involvement or occlusion, (V) contact with most proximal draining jejunal branch into $\operatorname{SMV}(2,11)$. By the AHPBA/SSAT/SSO, however, it is defined as: (I) no distant metastasis, (II) circumferential encasement of SMA and/or common hepatic artery (CHA), (III) abutment of CA due to tumor involvement, (IV) unreconstructable SMV and/or PV due to tumor involvement or occlusion [(12), reviewed in (18)]. Neoadjuvant chemotherapy, or rather more precisely: induction chemotherapy is a relatively novel concept for these tumors emerging from the encouraging results that were seen with FOLFIRINOX treatment in the palliative and adjuvant setting (19-22). More centers around the world are thus embracing multimodal pathways in the situation of questionable local resectability-and in unresectable LAPC there is nothing to lose: first line palliative chemotherapy treatment recommendations are FOLFIRINOX or gemcitabine/nab-paclitaxel and are thus identical to the regimen which would retrospectively be termed "induction chemotherapy" in case of a response that renders the patient suitable for surgical exploration $(1,23,24)$. In clinical reality this means that a patient with local involvement of visceral arteries but no signs of distant metastases who is judged unresectable by their surgeon will get sent for FOLFIRINOX treatment but also summoned for surgical reevaluation after at least 4-6, in some guidelines more cycles. In case of stable disease or better surgical exploration should be generously scheduled as data demonstrate secondary resectability in up to $60 \%$ of patients with imaging not accurately depicting local spread post chemotherapy $(25,26)$. Surgical evaluation will also consider CA 19-9 dynamics which have been shown to correlate with resectability $(27,28)$ and up to 12 preoperative cycles will usually be administered; in rare cases patients are resected after even more. Before and alongside FOLFIRINOX multiple (radio)chemotherapy regimens for LAPC have been proposed and evaluated in clinical trials (18). Some authors advocate for a cautious surgical approach as the importance of secondary resection in case of response or stable disease is still under debate to date, but other data have shown an advantage compared to continued chemotherapy (29-32).

Two 2016 meta-analyses on 365 and 355 patients, respectively $(19,33)$, found a median survival of 24.2 months with a pooled proportion of $25.9 \% / 28 \%$ of patients undergoing secondary resection after FOLFIRINOX and a range of $0-44 \%$. Resection rates up to $60 \%$ have been reported, though (26). Thus, in overall more than $70 \%$ of patients after induction chemotherapy the tumor is not suited to be resected. Often, this is due to distant metastasis at restaging or exploratory laparotomy, but a large proportion remains unresectable due to local spread. These patients will usually keep on receiving chemotherapy, maybe switched to second line agents, and are labelled palliative. Depending on the local standards regarding restagings during palliative chemotherapy they might be reevaluated for surgery again down the line but only rarely will undergo resection; international guidelines are lacking for this situation. If other methods of local tumor control for LAPC equal to surgery or at least superior to palliative 
chemotherapy were available this could open up new treatment pathways for LAPC patients.

\section{Ablation techniques pancreatic experience}

In contrast to other solid organ malignancies locally ablative strategies have not had much relevance for PDAC in the past as, in some series, major postinterventional morbidity and mortality have been reported due to anatomical features unique to the pancreas. Available data have not generated sufficient evidence to date to incorporate ablative techniques into any treatment guideline, yet, on an individual basis their application can be justified at specialized centers. Apart from possible benefits for oncological outcome, prevention of local complications of tumor growth and pain relief, some studies also point towards advantageous systemic immunological effects secondary to ablative treatment (34-37).

Although they encompass completely different therapeutic mechanisms, photodynamic therapy (PDT), brachytherapy, stereotactic body radiation (SBRT) and irreversible electroporation (IRE) are all non-thermal ablation techniques. This distinction is especially important for application on pancreatic lesions, as the surrounding anatomical structures, namely the duodenum, bile duct, mesentericoportal vessels and visceral arteries, are temperature sensitive and damage can lead to fatal complications. Techniques based on thermal tissue destruction include high-intensity focused ultrasound (HIFU), cryoablation, radiofrequency ablation (RFA), microwave ablation (MWA) and laser-induced thermotherapy (LITT). Whereas HIFU uses extracorporeal directed ultrasound waves focused on the target region and represents a non-invasive technique guided by ultrasound or magnetic resonance imaging (MRI), all other thermal ablative techniques involve placing a probe in the target tissue, either during laparotomy or percutaneously guided by crosssectional imaging or ultrasound; alternatively, endoscopic application has been successfully performed, too (38). After HIFU PV thrombosis, jaundice, pancreatic fistula, pancreatitis, GI bleeding, bowel perforation and severe skin burns have been described with a morbidity rate ranging from $0 \%$ to $23.2 \%$ [reviewed in (39)], and the technique has mostly been evaluated for pain palliation in pancreatic cancer $(40,41)$. Studies are often small, non-controlled, heterogeneous by population and protocols and no reliable survival advantages have been reported.

Cryoablation is a modality which can be applied during open surgery, by percutaneous needle insertion or endoscopically (42). The probe is rapidly cooled down to $-160{ }^{\circ} \mathrm{C}$ and reheated to $0{ }^{\circ} \mathrm{C}$ causing necrosis as well as apoptosis of the target zone; different protocols exist, but several freeze-thaw cycles are repeated. While it is rarely studied in Western countries, Chinese doctors seem to be more routinely using it and a consensus statement on pancreatic cryotherapy was issued with mostly Asian participation (43). However, the evidence to support the technique is still weak: In two retrospective studies comparing palliative surgery with and without additional cryoablation in overall 260 patients no survival benefit was found $(44,45)$. The risk profile was comparable to other thermal techniques on the pancreas with cases of pancreatic and biliary leakage, delayed gastric emptying and intestinal hemorrhage. Similar to the data for HIFU in LAPC, cryoablation can induce lasting pain control (46), but apart from the two studies mentioned above only case series are available for pancreatic indications. Addition of different cryo-adjuvants has been tested to achieve sufficient effects at the freeze zone margin (47) and postulated abscopal immunologic effects have led to combination treatment protocols including immunotherapies, especially in renal cell cancer and non-small cell lung cancer [reviewed in (48) but also PDAC $(37,49)]$.

In RFA, a high frequency alternating current produces heat leading to protein denaturation and necrosis starting at $60^{\circ} \mathrm{C}$ and vaporization and carbonization of the tissue beyond $100{ }^{\circ} \mathrm{C}(50)$. It is widely used on liver lesions and some other malignancies and thus available at more centers than other ablative techniques. Its limitations include a heat-sink effect, when thermal energy dissipates via the blood stream in the proximity of larger blood vessels, and a required safety distance of at least $5 \mathrm{~mm}$ to bile duct, vessels and the duodenum with some interventionalists preferring $10-15 \mathrm{~mm}$. RFA is most effective in symmetric, well defined lesions, whereas LAPC is known for its blurred boundaries. Most published studies report pancreatic RFA during laparotomy, however, endosonographic and percutaneous application has been reported in mostly experimental or exploratory settings, too (38). A 2014 systematic review on its use in LAPC found five studies on 158 patients and a reported median overall survival (OS) after RFA of 3-33 months, a morbidity related to RFA of $4-37 \%$, mortality of $0-19 \%$ and overall morbidity of $10-43 \%$ (51). In more recent publications, complication rates were improved: In several studies from Verona, Italy, (one percutaneous, six open surgery, one endoscopic) and one from Utrecht, Netherlands, OS ranged between 14.7 and 25.6 months [reviewed in $(37,39)$ ] with a severe 
complication rate of 0 to $28 \%$ and 30 -day mortality between 0 and $3 \%$. Complications included hepatic failure, duodenal perforation, severe acute pancreatitis, duodenal hemorrhage, pancreatic fistula, portal vein thrombosis, duodenal/biliary injury, gastric ulcer or fistula, hemoperitoneum. No randomized controlled trials are available on RFA in LAPC yet; the Dutch randomized controlled PELICAN trial was designed to close this gap, but no results have been published to date (Dutch trial register number NTR5517). Best available evidence on RFA in LAPC comes from prospective cohort studies without oncological control groups thus far.

MWA is a regularly used technique on hepatic lesions but has rarely been evaluated for pancreatic tumors. Microwaves cause heat by oscillation of predominantly $\mathrm{H}_{2} \mathrm{O}$ molecules in an electromagnetic field (52). Compared to its main contestant in hepatic application, RFA, MWA is faster with larger ablation volumes and less interference by heatsink effect, but also less predictable, pear-shaped ablation areas $(53,54)$. In pancreatic cancer, a total of 30 patients from case series have been reported in the literature with either percutaneous or open MWA (55-58). Apart from one patient with severe pancreatitis/pseudocyst and one with pseudoaneurysm of the GDA (56) no major complications were described; reported survival data are patchy and do not allow any conclusions as to oncological effectiveness.

LITT has to date most often been used in hepatic (59), intracranial (60), thyroid $(61,62)$ and prostate $(63)$ tumors. LITT operates with very fine needles and optical fibers and has a theoretical advantage of being less invasive and more precise than other ablative techniques, though comparative studies are lacking. In the only clinical report to date, Di Matteo et al. presented a case series of nine patients with localized PDAC unresponsive to chemotherapy and treated with EUS-guided LITT (64). No complete tumor ablation was intended, and no side effects were observed, though pending further clinical studies these data represent hardly more than a proof of principle.

PDT is closely related to LITT as it uses light wave energy as well, but the ablative effect is achieved by the interaction of the light waves with a photosensitizer which needs to be administered before. The chemical reaction produces radical oxygen species (ROS) which destroy surrounding tissue making it a non-thermal technique; depending on the selectivity of the agent for cancerous cells healthy tissue is spared. As light wave penetration is very shallow PDT has so far mainly been applied to intraluminal early stage malignancies such as esophageal, gastric, colorectal and bladder cancer [reviewed in (65)]. The dense peritumoral stroma in PDAC and its oxygen-deprived environment further limit light penetration and effectiveness of PDT. Since 2002, overall 44 patients receiving PDT for PDAC have been reported in the literature. Bown et al. treated 16 LAPC with meso-tetrahydroxyphenylchlorin and percutaneous, CT-guided PDT. Three duodenal obstructions and two gastroduodenal artery bleeds were observed; median OS was 9,5 months (66). Percutaneous PDT with verteporfin application resulted in a median OS of 8.8 months for 15 patients, no SAE were observed (67); and one patient is reported after use of Photolon ${ }^{\circledR}$ with PDT via EUS without SAE (68). In the most relevant study to date, DeWitt et al. gave 12 treatment-naïve LAPC patient porfimer sodium and PDT via EUS, followed by gemcitabine/nab-paclitaxel, with a median OS of 11.5 months. Notably, two patients underwent secondary resections with one complete pathological response; sunburns and hyperpigmentation were the reported side effects (69). Research in this field is focusing on the development of ideal photosensitizers; it is generally agreed that PDT is hardly a radical treatment but more of an adjunct in the strive for local control and palliation.

For brachytherapy radionuclides of phosphorus-32, iodine, gold, iridium, and yttrium have been studied for local PDAC treatment since the 1970ies with I-125 nuclides being the most frequently used agent (70). The radioactive seeds are implanted with specialized cannulas into the tumor under CT-, MRI- or EUS-guidance, but intraoperative use has been described, too (71). I-125 seeding has most often been studied in addition to other therapies and in case series, but a few contemporary, prospective comparative studies are available, too. One study comparing 66 unresectable patients receiving enteric bypass plus I-125 seeds or bypass alone for LAPC found significantly improved median OS of $11 \mathrm{vs} .7$ months in the brachytherapy group; pain scores were significantly better as well (72). Risks include pancreatitis, infection, seed migration and intestinal perforation $(73,74)$.

SBRT, also called stereotactic ablative body radiation ( $\mathrm{SABR}$ ), is a highly focused percutaneous radiation treatment with an intense dose of radiation on the target region, while limiting the dose to the surrounding organs, performed within few (usually 1-5) sessions at high doses (6-25 Gy). Cyberknife ${ }^{\circledR}$ is a specific technique of SBRT using real-time tracking of gold fiducial markers, realtime respiratory modelling and continuous monitoring to 
correct for tumor movement and radiation administered via a robotic arm. Only these highly accurate technologies achieve a sufficient, ablative biologically effective dose. Limitations of ablative dose delivery to pancreatic tumors are mostly due to the proximity of tumors to the duodenum, jejunum, and stomach, and trials examining conventional radiotherapy have largely failed to show any advantages $(3,75)$. Despite unconvincing data on conventional radiotherapy and limited evidence for SBRT current NCCN and ASCO guidelines recommend either for LAPC without distant progression after 6 months of chemotherapy $(2,24)$. A 2016 systematic review on SBRT in LAPC (76) found 19 studies (6 prospective, 13 retrospective, no RCTs) with a median OS ranging from 5.7 to 47 months; a more recent review on a partly overlapping group of studies gave a median OS after SBRT of 6.2 to 12.5 months and secondary resectability of $0-20.3 \%$ (39). Local complications reported include gastroparesis, gastrointestinal (duodenal) bleeding, duodenal or gastric outlet obstruction, duodenal perforation, gastric ulcer, anorexia, nausea/vomiting, and SMV/ inferior v. cava thrombosis (77) reported in few patients. Systemic radiation toxicity (cytopenias, nausea/diarrhea, infections) was reported in up to $28.5 \%$ of patients (39). RCTs are still lacking for SBRT in LAPC but some are currently ongoing: The US based RCT evaluating mFOLFIRINOX with and without SBRT, PANC0015, sponsored by Stanford University is not yet completed; so is the randomized controlled CROSSFIRE trial from the Netherlands, comparing IRE with SBRT. The CONKO-007 RCT from Germany, similar to PANC0015, evaluates the addition of radiation to induction therapy but no results have been published yet.

Since 2012, IRE, a non-thermal local tumor ablation technique, has been studied in LAPC (78). During IRE, tissue is destroyed by the application of a high-voltage direct current via electrodes inserted into the tumor, rendering cellular membranes porous and effectively breaking down the membrane barrier, thus killing the cell by predominantly inducing apoptosis (79). Notably, as there is only very little thermal effect, adjacent structures are spared. IRE can be applied on the pancreas via laparotomy or percutaneously by image-guided puncture; the most commonly used system is the NanoKnife ${ }^{\circledast}$ device. Several contraindications are usually applied: Tumor size $>5 \mathrm{~cm}$, combined severe stenosis of the CHA and PV given the $10 \%$ risk of an acute PV occlusion, as well as metal implants like bile duct stents/ cardiac pacemakers and a history of cardiac arrhythmias are considered incompatible with IRE, though the risk of metal implants is still being discussed (80). A 2017 systematic review (81) examined 10 studies of IRE in LAPC reporting on 446 patients and concluded that IRE is safe and feasible, but effects on survival were difficult to evaluate as the studies were heterogeneous in their designs and techniques. In an even more recent systematic review on 18 studies (all singlecenter, 8 retrospective, 7 prospective and 3 case series) the findings were similar; additionally, open IRE as compared to percutaneous had higher morbidity (35.6\%) and mortality (4.6\%) with 11 reported deaths attributed to GI bleeding, intraperitoneal hemorrhage, extensive bile duct and duodenal ischemia, liver failure, pulmonary embolism, multi-organ failure and unknown reasons (82). Overall, $5.3 \%$ of patients underwent secondary resection after downstaging post IRE, and median overall survival as reported from date of IRE ranged from 7-27 months. This systematic review included 498 patients overall even though it excluded IRE for margin accentuation during surgery and studies on IRE in combination therapies other than (radio-) chemotherapy. Another systematic review additionally found an overall complication rate of $30 \%$, with up to $59 \%$ in the rarely studied laparoscopic application of IRE, and associated mortality of $3 \%$ (83). In a current retrospective comparison of FOLFIRINOX with and without addition of IRE van Veldhuisen et al. found significantly longer OS (17.2 vs. 12.4 months) after added IRE (84), and in a matched analysis of 32 pairs receiving IRE or conventional radiotherapy after induction therapy IRE showed doubled median OS of 21.6 vs. 10.6 months (85). However, the prospective IMPALA study did not find improved median OS by addition of open IRE (16 months) to palliative chemotherapy (15 months), while underlining the importance of resection after induction therapy (34 months) (86). The prospective cohort study PANFIRE-II reported a median OS of 9/11 months after FOLFIRINOX/other chemotherapy and percutaneous IRE in 40 patients (87). To date, no randomized controlled data are available on IRE in LAPC; the ongoing CROSSFIRE study randomizing for FOLFIRINOX followed by either SBRT or IRE has not been published yet (NCT02791503). A few reports exist on immunological effects of IRE in humans pointing to suitability for combination with immunotherapies $(34,37,88,89)$.

\section{Discussion}

For all we know today, LAPC "only steel can heal": the most robust available data comparing LAPC resected after induction (radio-)chemotherapy with unresected LAPC 
demonstrate almost a doubling of median survival times [35.3 vs. 19.9 months in a matched analysis (29)] and a 5-year OS of $25.4 \%$ after secondary resection (32). In the light of these numbers, even if they are retrospective and include in part disseminated patients in the control groups, it seems highly unlikely that any future randomized controlled trials on resection versus continued (radio-) chemotherapy after response to induction therapy can be ethically justified and thus will probably never come. Only 20-30\% of all LAPC patients in the aforementioned collectives ultimately were able to undergo resection after induction treatment, but the true rate of resectable versus locally unresectable versus disseminated LAPC at reassessment after induction therapy has not been established so far as reports have been focusing on other aspects. Furthermore, no biomarkers are currently available to distinguish any of these LAPC subgroups. Though molecular pathology might lead to improved surgical treatment stratification in the future $(90,91)$, how can we best treat for the time being the remaining nondisseminated LAPC patients who, even under the most aggressive surgical approach, never get their tumor excised? Do we have local treatment options besides surgery to improve survival for this group? Reviewing the data the locally ablative techniques have yielded so far, possibly IRE currently has the highest potential to be included in future guidelines due to large patient series and the advantages of its non-thermal mode of action. Overall, though, the level of evidence generated by the studies on most ablative techniques is disappointing; controlled data are scarce, RCTs a rarity. As some trials with potentially high levels of evidence are still ongoing, the quality of data might improve in the near future, but currently there are modalities (MWA, LITT, PDT) that have been rarely studied and others (cryoablation, RFA, brachytherapy, HIFU, SBRT, IRE) with a sizeable number of publications, yet in both groups not a lot of evidence can be drawn. Clinically, this means that even though some techniques seem to be rather common they are performed on an exploratory/compassionate basis, and it will depend on local options if a patient is offered an ablative treatment and which, and under which protocol. Nevertheless, most data point towards acceptable safety and feasibility of the reviewed techniques with potential advantageous oncological effects. We therefore believe that high-quality trials are urgently needed to evaluate these modalities further. The first step, however, should be the definition of the patient subgroup who might benefit from non-surgical local treatment options. Considering the data on feasibility of extended resections and benefit of survival after resection in LAPC we call for the definition of a new PDAC subgroup: "Inconvertible LAPC". This concept should be used to characterize LAPC staged and treated with induction therapy according to consensus standards, followed by surgical (re-) exploration at centers equipped for extended resections which are judged locally unresectable by an experienced HPB surgeon. Alternatively, a multidisciplinary assessment after induction therapy that exploration is considered futile but dissemination is excluded is required.

The discussion about resectability of PDAC and the definition of BRPC and LAPC criteria has brought new standards to the reporting of surgical as well as oncological studies on pancreatic cancer and ensures comparability of measures and outcomes as well as standardized evaluation of new treatment pathways. However, for ablative therapies to date no precise definition of the target patient group exists and thus many publications report on a mix of stage II-IV cancers, which in the light of the latest data on powerful induction chemotherapies and outcomes of conversion surgery does not seem appropriate. Only now that effective, harmonized treatment pathways for LAPC are emerging can this specific subgroup even be defined. Furthermore, many publications on ablative therapies are unclear about the multidisciplinary assessment of the study participants; studies are often carried out by dedicated interventionalists, from radiology, radiation oncology, nuclear medicine or gastroenterology, who might not always be involved in the full scheme of oncological decision making. By defining the aforementioned requirements for LAPC patients to be grouped as "Inconvertible LAPC" and thus eligible for a locally ablative approach a multidisciplinary assessment according to current consensus guidelines on LAPC is ensured-the goal being that no patient should be unduly dismissed for surgical exploration as well as optimizing transferability of study results. Ideally, this specification of a distinct patient group will also put it in the spotlight more and hopefully prompt more trials designed to generate robust evidence. The first steps to apply the concept of "Inconvertible LAPC" should include large prospective cohort studies to establish its true incidence after state-ofthe-art induction treatment and under an ambitious surgical approach at specialized centers. Ideally, molecular profiling would be performed in these patients as well, possibly pointing out markers with relevance to further treatment modalities other than just type of chemotherapy. To more quickly approximate these data, our group is currently designing a patient-level meta-analysis focusing on the 
patient fraction already reported in the literature who fulfills these criteria. As reviewed here, published cohorts to date typically encompass a very heterogeneous population and tend to sum up different biological behaviour like local progression or dissemination as "unresectable". If more granular data could be extracted we might be able to put our finger on the special subgroup of "inconvertible LAPC". Future trials should then be designed in a way that outcomes of local ablation therapies can be directly compared to those of conversion surgery after induction as well as to palliative standards of care according to current guidelines. IRE and SBRT appear the most likely candidates here, as both come with the largest body of data for reference.

Even though some of the reviewed studies suggest the inherent superiority of an interventional, less-invasive approach over radical surgery, only if ablative strategies can convince under these standardized circumstances might it be time to compete against radical extended surgery in LAPC - the road for locally ablative techniques to replace surgery in LAPC is still very long.

\section{Acknowledgments}

Funding: None.

\section{Footnote}

Provenance and Peer Review: This article was commissioned by the Guest Editor (Elena Rangelova) for the series "Surgery for Locally Advanced Pancreatic Cancer" published in Fournal of Gastrointestinal Oncology. The article has undergone external peer review.

Conflicts of Interest: Both authors have completed the ICMJE uniform disclosure form (available at http://dx.doi. org/10.21037/jgo-20-379). The series "Surgery for Locally Advanced Pancreatic Cancer" was commissioned by the editorial office without any funding or sponsorship. The authors have no other conflicts of interest to declare.

Ethical Statement: Both authors are accountable for all aspects of the work in ensuring that questions related to the accuracy or integrity of any part of the work are appropriately investigated and resolved.

Open Access Statement: This is an Open Access article distributed in accordance with the Creative Commons
Attribution-NonCommercial-NoDerivs 4.0 International License (CC BY-NC-ND 4.0), which permits the noncommercial replication and distribution of the article with the strict proviso that no changes or edits are made and the original work is properly cited (including links to both the formal publication through the relevant DOI and the license). See: https://creativecommons.org/licenses/by-nc-nd/4.0/.

\section{References}

1. Ducreux M, Cuhna AS, Caramella C, et al. Cancer of the pancreas: ESMO Clinical Practice Guidelines for diagnosis, treatment and follow-up. Ann Oncol 2015;26 Suppl 5:v56-68.

2. Tempero MA, Malafa MP, Al-Hawary M, et al. Pancreatic Adenocarcinoma, Version 2.2017, NCCN Clinical Practice Guidelines in Oncology. J Natl Compr Canc Netw 2017;15:1028-61.

3. Grossberg AJ, Chu LC, Deig CR, et al. Multidisciplinary standards of care and recent progress in pancreatic ductal adenocarcinoma. CA Cancer J Clin 2020;70:375-403.

4. Versteijne E, Suker M, Groothuis K, et al. Preoperative Chemoradiotherapy Versus Immediate Surgery for Resectable and Borderline Resectable Pancreatic Cancer: Results of the Dutch Randomized Phase III PREOPANC Trial. J Clin Oncol 2020;38:1763-73.

5. Versteijne E, Vogel JA, Besselink MG, et al. Meta-analysis comparing upfront surgery with neoadjuvant treatment in patients with resectable or borderline resectable pancreatic cancer. Br J Surg 2018;105:946-58.

6. Cloyd JM, Heh V, Pawlik TM, et al. Neoadjuvant Therapy for Resectable and Borderline Resectable Pancreatic Cancer: A Meta-Analysis of Randomized Controlled Trials. J Clin Med 2020;9:1129.

7. Ye M, Zhang Q, Chen Y, et al. Neoadjuvant chemotherapy for primary resectable pancreatic cancer: a systematic review and meta-analysis. HPB (Oxford) 2020;22:821-32.

8. Strobel O, Lorenz P, Hinz U, et al. Actual Five-year Survival After Upfront Resection for Pancreatic Ductal Adenocarcinoma: Who Beats the Odds? Ann Surg 2020. [Epub ahead of print].

9. Conroy T, Desseigne F, Ychou M, et al. FOLFIRINOX versus gemcitabine for metastatic pancreatic cancer. $\mathrm{N}$ Engl J Med 2011;364:1817-25.

10. Isaji S, Mizuno S, Windsor JA, et al. International consensus on definition and criteria of borderline resectable pancreatic ductal adenocarcinoma 2017. Pancreatology 2018;18:2-11. 
11. Bockhorn M, Uzunoglu FG, Adham M, et al. Borderline resectable pancreatic cancer: a consensus statement by the International Study Group of Pancreatic Surgery (ISGPS). Surgery 2014;155:977-88.

12. Callery MP, Chang KJ, Fishman EK, et al. Pretreatment assessment of resectable and borderline resectable pancreatic cancer: expert consensus statement. Ann Surg Oncol 2009;16:1727-33.

13. Katz MH, Pisters PW, Evans DB, et al. Borderline resectable pancreatic cancer: the importance of this emerging stage of disease. J Am Coll Surg 2008;206:83346; discussion 846-8.

14. Varadhachary GR, Tamm EP, Abbruzzese JL, et al. Borderline resectable pancreatic cancer: definitions, management, and role of preoperative therapy. Ann Surg Oncol 2006;13:1035-46.

15. Kaufmann B, Hartmann D, D'Haese JG, et al. Neoadjuvant Treatment for Borderline Resectable Pancreatic Ductal Adenocarcinoma. Dig Surg 2019;36:455-61.

16. Janssen QP, Buettner S, Suker M, et al. Neoadjuvant FOLFIRINOX in Patients With Borderline Resectable Pancreatic Cancer: A Systematic Review and Patient-Level Meta-Analysis. J Natl Cancer Inst 2019;111:782-94.

17. Hackert T. Surgery for Pancreatic Cancer after neoadjuvant treatment. Ann Gastroenterol Surg 2018;2:413-8.

18. He J, Page AJ, Weiss M, et al. Management of borderline and locally advanced pancreatic cancer: where do we stand? World J Gastroenterol 2014;20:2255-66.

19. Rombouts SJ, Walma MS, Vogel JA, et al. Systematic Review of Resection Rates and Clinical Outcomes After FOLFIRINOX-Based Treatment in Patients with Locally Advanced Pancreatic Cancer. Ann Surg Oncol 2016;23:4352-60.

20. Dhir M, Malhotra GK, Sohal DPS, et al. Neoadjuvant treatment of pancreatic adenocarcinoma: a systematic review and meta-analysis of 5520 patients. World J Surg Oncol 2017;15:183.

21. Conroy T, Gavoille C, Samalin E, et al. The role of the FOLFIRINOX regimen for advanced pancreatic cancer. Curr Oncol Rep 2013;15:182-9.

22. Conroy T, Hammel P, Hebbar M, et al. FOLFIRINOX or Gemcitabine as Adjuvant Therapy for Pancreatic Cancer. N Engl J Med 2018;379:2395-406.

23. Wolfe AR, Prabhakar D, Yildiz VO, et al. Neoadjuvantmodified FOLFIRINOX vs nab-paclitaxel plus gemcitabine for borderline resectable or locally advanced pancreatic cancer patients who achieved surgical resection. Cancer Med 2020;9:4711-23.

24. Balaban EP, Mangu PB, Khorana AA, et al. Locally Advanced, Unresectable Pancreatic Cancer: American Society of Clinical Oncology Clinical Practice Guideline. J Clin Oncol 2016;34:2654-68.

25. Ferrone CR, Marchegiani G, Hong TS, et al. Radiological and surgical implications of neoadjuvant treatment with FOLFIRINOX for locally advanced and borderline resectable pancreatic cancer. Ann Surg 2015;261:12-7.

26. Hackert T, Sachsenmaier M, Hinz U, et al. Locally Advanced Pancreatic Cancer: Neoadjuvant Therapy With Folfirinox Results in Resectability in $60 \%$ of the Patients. Ann Surg 2016;264:457-63.

27. Boone BA, Steve J, Zenati MS, et al. Serum CA 19-9 response to neoadjuvant therapy is associated with outcome in pancreatic adenocarcinoma. Ann Surg Oncol 2014;21:4351-8.

28. Heger U, Sun H, Hinz U, et al. Induction chemotherapy in pancreatic cancer: CA 19-9 may predict resectability and survival. HPB (Oxford) 2020;22:224-32.

29. Gemenetzis G, Groot VP, Blair AB, et al. Survival in Locally Advanced Pancreatic Cancer After Neoadjuvant Therapy and Surgical Resection. Ann Surg 2019;270:340-7.

30. Tsai S, Christians KK, Ritch PS, et al. Multimodality Therapy in Patients With Borderline Resectable or Locally Advanced Pancreatic Cancer: Importance of Locoregional Therapies for a Systemic Disease. J Oncol Pract 2016;12:915-23.

31. van Veldhuisen E, van den Oord C, Brada LJ, et al. Locally Advanced Pancreatic Cancer: Work-Up, Staging, and Local Intervention Strategies. Cancers (Basel) 2019;11:976.

32. Rangelova E, Wefer A, Persson S, et al. Surgery Improves Survival After Neoadjuvant Therapy for Borderline and Locally Advanced Pancreatic Cancer: A Single Institution Experience. Ann Surg 2021;273:579-86.

33. Suker M, Beumer BR, Sadot E, et al. FOLFIRINOX for locally advanced pancreatic cancer: a systematic review and patient-level meta-analysis. Lancet Oncol 2016;17:801-10.

34. Scheffer HJ, Stam AGM, Geboers B, et al. Irreversible electroporation of locally advanced pancreatic cancer transiently alleviates immune suppression and creates a window for antitumor $\mathrm{T}$ cell activation. Oncoimmunology 2019;8:1652532.

35. Napoletano C, Taurino F, Biffoni M, et al. RFA strongly modulates the immune system and anti-tumor immune responses in metastatic liver patients. Int J Oncol 
2008;32:481-90.

36. Al-Sakere B, Bernat C, Andre F, et al. A study of the immunological response to tumor ablation with irreversible electroporation. Technol Cancer Res Treat 2007;6:301-6.

37. Geboers B, Ruarus AH, Nieuwenhuizen S, et al. Needle-guided ablation of locally advanced pancreatic cancer: cytoreduction or immunomodulation by in vivo vaccination? Chin Clin Oncol 2019;8:61.

38. Testoni SGG, Healey AJ, Dietrich CF, et al. Systematic review of endoscopy ultrasound-guided thermal ablation treatment for pancreatic cancer. Endosc Ultrasound 2020;9:83-100.

39. Ruarus A, Vroomen L, Puijk R, et al. Locally Advanced Pancreatic Cancer: A Review of Local Ablative Therapies. Cancers (Basel) 2018;10:16.

40. Dababou S, Marrocchio C, Rosenberg J, et al. A metaanalysis of palliative treatment of pancreatic cancer with high intensity focused ultrasound. J Ther Ultrasound 2017;5:9.

41. Vidal-Jove J, Perich E, Del Castillo MA. Ultrasound Guided High Intensity Focused Ultrasound for malignant tumors: The Spanish experience of survival advantage in stage III and IV pancreatic cancer. Ultrason Sonochem 2015;27:703-6.

42. Arcidiacono PG, Carrara S, Reni M, et al. Feasibility and safety of EUS-guided cryothermal ablation in patients with locally advanced pancreatic cancer. Gastrointest Endosc 2012;76:1142-51.

43. He L, Niu L, Korpan NN, et al. Clinical Practice Guidelines for Cryosurgery of Pancreatic Cancer: A Consensus Statement From the China Cooperative Group of Cryosurgery on Pancreatic Cancer, International Society of Cryosurgery, and Asian Society of Cryosurgery. Pancreas 2017;46:967-72.

44. Li J, Chen X, Yang H, et al. Tumour cryoablation combined with palliative bypass surgery in the treatment of unresectable pancreatic cancer: a retrospective study of 142 patients. Postgrad Med J 2011;87:89-95.

45. Song ZG, Hao JH, Gao S, et al. The outcome of cryoablation in treating advanced pancreatic cancer: a comparison with palliative bypass surgery alone. J Dig Dis 2014;15:561-9.

46. Ferrer-Mileo L, Luque Blanco AI, Gonzalez-Barboteo J. Efficacy of Cryoablation to Control Cancer Pain: A Systematic Review. Pain Pract 2018;18:1083-98.

47. Baust JG, Snyder KK, Santucci KL, et al. Cryoablation: physical and molecular basis with putative immunological consequences. Int J Hyperthermia 2019;36:10-6.

48. Aarts BM, Klompenhouwer EG, Rice SL, et al. Cryoablation and immunotherapy: an overview of evidence on its synergy. Insights Imaging 2019;10:53.

49. Petrou A, Moris D, Paul Tabet P, et al. Ablation of the locally advanced pancreatic cancer: An introduction and brief summary of techniques. J BUON 2016;21:650-8.

50. Paiella S, Salvia R, Ramera M, et al. Local Ablative Strategies for Ductal Pancreatic Cancer (Radiofrequency Ablation, Irreversible Electroporation): A Review. Gastroenterol Res Pract 2016;2016:4508376.

51. Fegrachi S, Besselink MG, van Santvoort HC, et al. Radiofrequency ablation for unresectable locally advanced pancreatic cancer: a systematic review. HPB (Oxford) 2014;16:119-23.

52. Simon CJ, Dupuy DE, Mayo-Smith WW. Microwave ablation: principles and applications. Radiographics 2005;25 Suppl 1:S69-83.

53. Poulou LS, Botsa E, Thanou I, et al. Percutaneous microwave ablation vs radiofrequency ablation in the treatment of hepatocellular carcinoma. World J Hepatol 2015;7:1054-63.

54. Glassberg MB, Ghosh S, Clymer JW, et al. Microwave ablation compared with radiofrequency ablation for treatment of hepatocellular carcinoma and liver metastases: a systematic review and meta-analysis. Onco Targets Ther 2019;12:6407-38.

55. Lygidakis NJ, Sharma SK, Papastratis P, et al. Microwave ablation in locally advanced pancreatic carcinoma--a new look. Hepatogastroenterology 2007;54:1305-10.

56. Carrafiello G, Ierardi AM, Fontana F, et al. Microwave ablation of pancreatic head cancer: safety and efficacy. J Vasc Interv Radiol 2013;24:1513-20.

57. Vogl TJ, Panahi B, Albrecht MH, et al. Microwave ablation of pancreatic tumors. Minim Invasive Ther Allied Technol 2018;27:33-40.

58. Ierardi AM, Biondetti P, Coppola A, et al. Percutaneous microwave thermosphere ablation of pancreatic tumours. Gland Surg 2018;7:59-66.

59. Vogl TJ, Farshid P, Naguib NN, et al. Thermal ablation of liver metastases from colorectal cancer: radiofrequency, microwave and laser ablation therapies. Radiol Med 2014;119:451-61.

60. Holste KG, Orringer DA. Laser interstitial thermal therapy. Neurooncol Adv 2019;2:vdz035.

61. Ritz JP, Lehmann KS, Schumann T, et al. Effectiveness of various thermal ablation techniques for the treatment of nodular thyroid disease--comparison of laser-induced 
thermotherapy and bipolar radiofrequency ablation. Lasers Med Sci 2011;26:545-52.

62. Pacella CM, Mauri G, Achille G, et al. Outcomes and Risk Factors for Complications of Laser Ablation for Thyroid Nodules: A Multicenter Study on 1531 Patients. J Clin Endocrinol Metab 2015;100:3903-10.

63. Castellani D, Pirola GM, Pacchetti A, et al. State of the Art of Thulium Laser Enucleation and Vapoenucleation of the Prostate: A Systematic Review. Urology 2020;136:19-34.

64. Di Matteo FM, Saccomandi P, Martino M, et al. Feasibility of EUS-guided Nd:YAG laser ablation of unresectable pancreatic adenocarcinoma. Gastrointest Endosc 2018;88:168-74.e1.

65. Wang Y, Wang H, Zhou L, et al. Photodynamic therapy of pancreatic cancer: Where have we come from and where are we going? Photodiagnosis Photodyn Ther 2020;31:101876.

66. Bown SG, Rogowska AZ, Whitelaw DE, et al. Photodynamic therapy for cancer of the pancreas. Gut 2002;50:549-57.

67. Huggett MT, Jermyn M, Gillams A, et al. Phase I/II study of verteporfin photodynamic therapy in locally advanced pancreatic cancer. Br J Cancer 2014;110:1698-704.

68. Choi JH, Oh D, Lee JH, et al. Initial human experience of endoscopic ultrasound-guided photodynamic therapy with a novel photosensitizer and a flexible laser-light catheter. Endoscopy 2015;47:1035-8.

69. DeWitt JM, Sandrasegaran K, O'Neil B, et al. Phase 1 study of EUS-guided photodynamic therapy for locally advanced pancreatic cancer. Gastrointest Endosc 2019;89:390-8.

70. Cazacu IM, Singh BS, Saftoiu A, et al. Endoscopic Ultrasound-Guided Treatment of Pancreatic Cancer. Curr Gastroenterol Rep 2020;22:27.

71. Wang J, Jiang Y, Li J, et al. Intraoperative ultrasoundguided iodine-125 seed implantation for unresectable pancreatic carcinoma. J Exp Clin Cancer Res 2009;28:88.

72. Zheng Z, Xu Y, Zhang S, et al. Surgical bypass and permanent iodine-125 seed implantation vs. surgical bypass for the treatment of pancreatic head cancer. Oncol Lett 2017;14:2838-44.

73. Lv WF, Lu D, Xiao JK, et al. The side effects and complications of percutaneous iodine- 125 seeds implantation under CT-guide for patients with advanced pancreatic cancer. Medicine (Baltimore) 2017;96:e9535.

74. Jia SN, Wen FX, Gong TT, et al. A review on the efficacy and safety of iodine-125 seed implantation in unresectable pancreatic cancers. Int J Radiat Biol 2020;96:383-9.

75. Hammel P, Huguet F, van Laethem JL, et al. Effect of Chemoradiotherapy vs Chemotherapy on Survival in Patients With Locally Advanced Pancreatic Cancer Controlled After 4 Months of Gemcitabine With or Without Erlotinib: The LAP07 Randomized Clinical Trial. JAMA 2016;315:1844-53.

76. Petrelli F, Comito T, Ghidini A, et al. Stereotactic Body Radiation Therapy for Locally Advanced Pancreatic Cancer: A Systematic Review and Pooled Analysis of 19 Trials. Int J Radiat Oncol Biol Phys 2017;97:313-22.

77. Rombouts SJ, Vogel JA, van Santvoort HC, et al. Systematic review of innovative ablative therapies for the treatment of locally advanced pancreatic cancer. Br J Surg 2015;102:182-93.

78. Martin RC, 2nd, McFarland K, Ellis S, et al. Irreversible electroporation therapy in the management of locally advanced pancreatic adenocarcinoma. J Am Coll Surg 2012;215:361-9.

79. Davalos RV, Mir IL, Rubinsky B. Tissue ablation with irreversible electroporation. Ann Biomed Eng 2005;33:223-31.

80. Timmer FEF, Geboers B, Ruarus AH, et al. Irreversible Electroporation for Locally Advanced Pancreatic Cancer. Tech Vasc Interv Radiol 2020;23:100675.

81. Ansari D, Kristoffersson S, Andersson R, et al. The role of irreversible electroporation (IRE) for locally advanced pancreatic cancer: a systematic review of safety and efficacy. Scand J Gastroenterol 2017;52:1165-71.

82. Moris D, Machairas N, Tsilimigras DI, et al. Systematic Review of Surgical and Percutaneous Irreversible Electroporation in the Treatment of Locally Advanced Pancreatic Cancer. Ann Surg Oncol 2019;26:1657-68.

83. Lafranceschina S, Brunetti O, Delvecchio A, et al. Systematic Review of Irreversible Electroporation Role in Management of Locally Advanced Pancreatic Cancer. Cancers (Basel) 2019;11:1718.

84. van Veldhuisen E, Vroomen LG, Ruarus AH, et al. Value of CT-Guided Percutaneous Irreversible Electroporation Added to FOLFIRINOX Chemotherapy in Locally Advanced Pancreatic Cancer: A Post Hoc Comparison. J Vasc Interv Radiol 2020;31:1600-8.

85. He C, Wang J, Sun S, et al. Irreversible electroporation versus radiotherapy after induction chemotherapy on survival in patients with locally advanced pancreatic cancer: a propensity score analysis. BMC Cancer 2019;19:394.

86. Vogel JA, Rombouts SJ, de Rooij T, et al. Induction Chemotherapy Followed by Resection or Irreversible 
Electroporation in Locally Advanced Pancreatic Cancer (IMPALA): A Prospective Cohort Study. Ann Surg Oncol 2017;24:2734-43.

87. Ruarus AH, Vroomen L, Geboers B, et al. Percutaneous Irreversible Electroporation in Locally Advanced and Recurrent Pancreatic Cancer (PANFIRE-2): A Multicenter, Prospective, Single-Arm, Phase II Study. Radiology 2020;294:212-20.

88. Pandit H, Hong YK, Li Y, et al. Evaluating the Regulatory Immunomodulation Effect of Irreversible Electroporation (IRE) in Pancreatic Adenocarcinoma. Ann Surg Oncol 2019;26:800-6.

Cite this article as: Heger U, Hackert T. Can local ablative techniques replace surgery for locally advanced pancreatic cancer? J Gastrointest Oncol 2021;12(5):2536-2546. doi: 10.21037/ jgo-20-379
89. He C, Wang J, Sun S, et al. Immunomodulatory Effect after Irreversible Electroporation in Patients with Locally Advanced Pancreatic Cancer. J Oncol 2019;2019:9346017.

90. Aung KL, Fischer SE, Denroche RE, et al. GenomicsDriven Precision Medicine for Advanced Pancreatic Cancer: Early Results from the COMPASS Trial. Clin Cancer Res 2018;24:1344-54.

91. Wang Y, Park JYP, Pacis A, et al. A Preclinical Trial and Molecularly Annotated Patient Cohort Identify Predictive Biomarkers in Homologous Recombination-deficient Pancreatic Cancer. Clin Cancer Res 2020;26:5462-76. 